\title{
Treatment and Diagnostic Considerations in a Complex Psychiatric Case - a Case Report
}

\author{
Evan N. Caporaso M.D. \\ Naval Medical Center San Diego, caporasoevan@gmail.com \\ John M. Woo M.D. \\ Naval Medical Center San Diego, john.m.woo.mil@mail.mil
}

Cooper Rowan Medical Journal: https://rdw.rowan.edu/crjcsm

Would you like to be a reviewer? Please fill in this short form to express your interest.

\section{Recommended Citation}

Caporaso, Evan N. M.D. and Woo, John M. M.D. (2020) "Treatment and Diagnostic Considerations in a Complex Psychiatric Case - a Case Report," Cooper Rowan Medical Journal: Vol. 2 : Iss. 1 , Article 5. DOI: 10.31986/issn.2578-3343_vol2iss1.5

Available at: https://rdw.rowan.edu/crjcsm/vol2/iss1/5

\section{(c) (i)}

This work is licensed under a Creative Commons Attribution 4.0 License.

This Case Reports and Case Series is brought to you for free and open access by the Rowan University Journals at Rowan Digital Works. It has been accepted for inclusion in Cooper Rowan Medical Journal by an authorized editor of Rowan Digital Works. For more information, please contact brush@rowan.edu. 
Treatment and Diagnostic Considerations in a Complex Psychiatric Case - a Case Report 


\title{
Treatment and Diagnostic Considerations in a Complex Psychiatric Case - a Case Report
}

\author{
Evan N. Caporaso, M.D. ${ }^{1 *}$ \& John M. Woo, M.D. ${ }^{1}$ \\ ${ }^{1}$ Directorate for Mental Health, Naval Medical Center, San Diego, California, United States \\ *Corresponding author: caporasoevan@gmail.com (Evan N. Caporaso, M.D.)
}

\begin{abstract}
A patient with a history of autism spectrum disorder and epilepsy was hospitalized for management of acute onset psychosis and agitation. The acuity of his behaviors warranted abrupt shifts in treatment and multiple pharmacologic interventions were ineffective. The atypical nature of his presentation and intense pressure from ancillary staff to consider organic etiologies drove frequent transitions of care within the hospital setting. Multiple diagnoses were considered including a primary psychosis, excited catatonia and antiepileptic drug-induced psychotic disorder. Ultimately the patient was diagnosed with bipolar disorder and effectively treated with quetiapine and valproic acid. The authors suggest that rapid consideration of comorbid bipolar disorder in autism spectrum disorder patients presenting with affective dysregulation may expedite trial of an anticonvulsant with mood stabilizing properties, which would have simplified this patient's clinical course and limited potential for iatrogenic harm. This additional diagnosis and associated course of treatment should be considered when a history of epilepsy and autism spectral disorder is present with the symptom complex manifested in this patient.
\end{abstract}

\section{INTRODUCTION:}

Autism spectrum disorder (ASD) is a neurodevelopmental condition broadly characterized by deficits in social interaction and restrictive patterns of behavior ${ }^{1}$. Although prevalence estimates for ASD have varied across investigations, a 2014 study utilizing data from the Global Burden of Disease 2010 initiative estimated a global population prevalence of approximately 7.6 cases of ASD per 1000 persons, and established the importance of the condition as a major source of morbidity and disability within populations $^{2}$. On an individual level ASD is a spectrum condition with tremendous clinical heterogeneity, and mental health treatment of ASD is complicated by high rates of medical and psychiatric comorbidity. Epilepsy, for example, is present in approximately $20 \%$ of individuals with an $\mathrm{ASD}^{3}$. Within the domain 
of mental health, a comparative study of referrals to a pediatric psychopharmacology program demonstrated that individuals with ASD $(n=217)$ had an average of 6 comorbid psychiatric diagnoses ${ }^{4}$. Accordingly, the targets and intensities of treatment often vary between ASD individuals.

Severe behavioral problems sometimes occur as part of the constellation of symptoms associated with ASD. Classified under 'irritability' within ASD research literature, these disruptive behavioral problems are particularly distressing to families, caregivers and providers and often emerge as targets of clinical treatment. Suggestive of the scope of this problem, a multi-site research study in North America involving 1,380 children and adolescents with ASD found that $68 \%$ of patients had a history of physically aggressive behavior towards a caregiver ${ }^{5}$. The preponderance of research to-date on pharmacologic interventions for irritability in ASD has focused on dopamine antagonists, or antipsychotic class medications. Historically, the first generation (or "conventional") antipsychotic haloperidol was most widely employed for this purpose, though its contemporary use is more limited after studies demonstrated its association with high rates of adverse dyskinesias in ASD individuals ${ }^{6}$. Second generation (or "atypical") antipsychotics are now generally regarded as first-line agents for the irritability or 'severely disruptive behavior' symptom cluster in ASD, with risperidone and aripiprazole having specific FDA approvals for this indication ${ }^{7}$. Evidence for other antipsychotic class medications, including olanzapine and quetiapine, has been $\operatorname{mixed}^{7}$. An open-label trial in 2011 involving 11 patients with ASD demonstrated reductions in behavioral agitation with administration of low-dose Quetiapine ${ }^{8}$, though other studies have reported negative findings.

With regard to other psychotropic agents, research into the efficacy of valproic acid (VPA) in the treatment of ASD-related irritability was stimulated by a series of case reports which indicated that VPA-related treatment of epilepsy comorbid to ASD was associated with improvements in other neurodevelopmental symptom domains, in conjunction with a separate body of literature indicating the more general efficacy of VPA in amelioration of aggression. Overall, studies-to-date indicate that VPA may be effective in treating disruptive behavior associated with ASD, particularly in cases where ASD is associated with epilepsy and/or other affective disorders.

This case study describes a patient with ASD, active epilepsy, and severe episodic affective dysregulation whose clinical course was confounded by diagnostic uncertainty and notable for symptomatology recalcitrant to several psychotropic trials. His symptoms, which were ultimately favored to be etiologically related to comorbid bipolar disorder, remitted on a combination of VPA and quetiapine. The authors suggest that rapid consideration of comorbid bipolar disorder in ASD patients presenting with affective dysregulation may facilitate an expeditious trial of an anticonvulsant with mood stabilizing properties, which would have simplified this patient's clinical course and limited potential for iatrogenic harm. This 
course of treatment should especially be considered where a history of active epilepsy is present.

\section{PATIENT INFORMATION:}

The patient was a 22 year-old man who was brought to the emergency department by his parents for evaluation of multiple concerns including recent onset of psychosis, potential seizure activity and agitated behaviors at home. The patient had a history of an autism spectrum disorder, epilepsy and depression. The patient was diagnosed with autism spectrum disorder with borderline intellectual functioning at age 5 . Outside of episodes of depression, the patient had no other psychiatric history including no history of past hospitalization, suicidality, psychosis or aggression towards others. He had no known history of substance abuse or personal trauma. With family support, the patient was at baseline capable of completing tasks of daily living and maintained employment at a job allocated for individuals with disabilities.

Collateral history acquired from informants (family members) served as the only reliable source of information relating to his history of present illness and the patient's parents provided a complex and multifactorial recent history. Their story began 6 months ago, at which time the patient had been tapered off his anti-epileptic, valproic acid, following a seizure-free period of several years. Several months later the patient developed two generalized tonic-clonic seizures prompting initiation of topiramate. Shortly thereafter, approximately 1 month prior to his current presentation, the patient began exhibiting paranoia and marked irritable changes in his affect.

These changes in behavior led to interface with emergency services and two outside psychiatric hospitalizations for psychosis in the month preceding his current presentation. Over the course of these hospitalizations he was trialed on low-dose risperidone and quetiapine, in addition to replacement of topiramate with lacosamide, then re-initiation of valproic acid as his primary anti-epileptic. None of these interventions had been effective in ameliorating the patient's agitation or psychosis and the family presented to the emergency department in a state of ongoing crisis.

\section{CLINICAL FINDINGS:}

Initial evaluation with physical exam revealed ecchymosis across the chest and abdomen with extension to the upper and lower extremities. This was associated with tenderness to palpation but no crepitus, gross deformity or lacerating injuries. Neurologic exam did not reveal focal deficits, though assessment was limited due to the patient's distractibility and noncompliance. Heart and lung exam were normal and his airway was patent. A comprehensive laboratory workup was notable for creatine kinase to $3500 \mathrm{U} / \mathrm{L}$, liver enzyme abnormalities (ALT/AST 109/155 U/L), a valproate level of $62.9 \mathrm{ug} / \mathrm{mL}$ and cardiac troponins of $0.026 \mathrm{ng} / \mathrm{mL}$. All values were down trending on repeat assessment. A radiologic workup consisting of 2-view chest X-ray, non-contrast head CT and contrast CT of the chest were without any notable abnormalities. An EKG was without ST-segment changes. The patient remained significantly agitated and 
disruptive in the emergency department and received two doses of $5 \mathrm{mg}$ haloperidol, $2 \mathrm{mg}$ lorazepam and $50 \mathrm{mg}$ diphenhydramine with minimal improvement.

The patient exhibited threatening behavior in the emergency department and upon psychiatric ward admission. On his first day of admission he was placed on assault precautions and required several instances of locked seclusion and physical 4-point restraint. Antipsychotics were initially withheld due to a differential diagnosis which included Neuroleptic Malignant Syndrome and agitation secondary to extrapyramidal symptoms on the basis of the patient's mask-life facies, bradykinesia and rigidity. An agitated catatonia was also considered. Owing to ongoing laboratory evaluation which yielded elevated C-reactive protein, the differential diagnosis was broadened to include central nervous system infection or immune-mediated encephalopathy. Accordingly, the patient was transferred to the ICU for sedation and acquisition of CSF studies - these were normal, including an assay for anti-NMDA receptor antibodies.

\section{THERAPEUTIC INTERVENTIONS:}

Observation on the inpatient psychiatric ward yielded target symptomatology which consisted of cognitive disorganization, insomnia, a blunted but pressured affect, behavioral agitation, distractibility and profound irritability. The patient was additionally observed to be hallucinating. The patient was initiated on olanzapine but continued to exhibit a high tempo of behavioral dysregulation and minimal, fractured sleep. Given clinical non-response to olanzapine over 4 days of treatment, during which over $60 \mathrm{mg}$ of olanzapine was administered, in conjunction with persistent mental status exam manifestations of extrapyramidal symptoms, the decision was made to withhold further dopaminergic blockade and treat with oral benzodiazepines in conjunction with clonidine and his maintenance dose of valproic acid. A benzodiazepine challenge resulted in an initial positive response, but over subsequent days the patient's behavior again relapsed to a state of physical agitation and aggression without sustained improvement in any other domains of symptomatology. His extrapyramidal symptoms also did not remit.

Following several instances of injury to the patient's hospital attendants, as well as auxiliary staff burdens including constant 2-to-3 person watches warranted by the patient's highly disorganized behavior, a difficult case conference was held and attended by representatives of multiple disciplines. Ultimately, a plan was formulated which involved transfer to the ICU for dexmedatomidine sedation for medication washout, based on the concern that this developmentally delayed patient was being disinhibited by the rendered benzodiazepine treatments. A prolonged EEG was acquired during this wash-out period which was negative for any epileptiform changes.

The patient's symptoms were generally unimproved following the washout period and he was transferred back to the psychiatric ward. A previously closed section of the ward was re-opened, allowing the patient to be domiciled in an environment with less external stimuli and limited potential for harm towards other 
patients in the hospital.

The patient was initiated on aggressively titrated quetiapine and his valproic acid dose was aggressively increased from 1,000 to $2,000 \mathrm{mg}$ daily, resulting in a serum valproate level of $115.2 \mathrm{ug} / \mathrm{mL}$. Additionally, eslicarbazepine was added to his regimen after he exhibited a recurrence of seizure-like activity. On this regimen the patient's agitation and aggressive disorganized behavior gradually improved in a sustained manner. His extrapyramidal symptoms, until this point a constant feature of his mental status exam, resolved. After a complex hospitalization lasting almost three weeks, the patient was discharged home. FOLLOW UP

Although the patient had follow-up outside of our direct hospital system, a review of his electronic medical record verified ongoing and current prescription of quetiapine and valproic Acid. This is interpreted by the authors as a measure of adherence and tolerability of these medications.

\section{DISCUSSION}

Autism spectrum disorder (ASD) is a neurodevelopmental condition with significant medical and neuropsychiatric overlay, contributing to the potential for diagnostic and treatment challenges in the acute care setting. Although there are multiple studies investigating the use of antipsychotics and other agents for use in the treatment of behavioral agitation associated with ASD, there are fewer delineating the pathways of treatment when ASD is comorbid with severe mental illness - a comorbidity which may be more common than in the general population ${ }^{9}$.

An important feature of the patient's hospitalization were clinical concerns that his condition was being prolonged or exacerbated by iatrogenic means - for example, agitation as a result of induction of severe extrapyramidal symptoms associated with use of dopamine blocking agents. Akathisia in particular has been shown to worsen psychopathology and exacerbate impulsive and aggressive behavior ${ }^{10}$. The patient exhibited autonomic and motor abnormalities with diaphoresis, tremulousness, Parkinsonism and rigidity which did not respond to common interventions including withdrawal of antipsychotic therapy or treatment with anticholinergics (benztropine) or benzodiazepines. Laboratory markers of elevated creatine kinase and transaminitis, in conjunction with moments of urinary incontinence and other behaviors which were regressed from baseline, raised concern for possible neuroleptic malignant syndrome. However, the patient was afebrile for the duration of his hospital course and his vital signs remained stable during close longitudinal observation. His symptoms also did not respond meaningfully to a benzodiazepine challenge, as might be expected in an agitated catatonia.

The temporal relationship between initiation of a new anti-epileptic drug (AED) topiramate and onset of the patient's presenting symptomatology raised concern for antiepileptic drug-induced psychotic disorder 
(AIPD), a known phenomenon but one without rigorously defined diagnostic or classification criteria. Although initial clinical trial data did not identify a significant link between topiramate and anti-epileptic drug induced psychosis ${ }^{11}$, subsequent studies have provided evidence of a possible relationship ${ }^{12,13}$. However, in these cases of topiramate-induced psychosis, symptoms were noted to resolve rapidly with drug discontinuation (generally within 48 hours) and this was inconsistent with the patient's longer duration of illness. Given the overall high index of suspicion for an organic etiology of illness, a rare hyperammonemic encephalopathic condition related to co-treatment with topiramate and valproic acid was also considered, though subsequent laboratory studies did not support this diagnosis ${ }^{14}$. More recent investigations into the phenomena of antiepileptic-induced psychosis have identified female gender, temporal lobe involvement, a shorter duration of psychosis (less than 2 weeks) and use of levetiracetam as statistically significant correlates of risk for this condition ${ }^{15}$.

Case reports supporting use of valproic acid in individuals with ASD with affective dysregulation and irritability have led to interrogation of the clinical utility of this medicine through more rigorous study methodologies over time. J. Childs et al presented a case study of autistic twins treated with VPA for management of absence seizures, who then exhibited global improvement of ASD-related symptomatology ${ }^{16}$. A case series of 3 children with ASD and electroencephalographic changes, but no frank seizure disorder diagnoses, were noted to exhibit significant improvement in ASD-related behaviors with VPA treatment ${ }^{17}$. An additional case series involving VPA treatment of two patients with severe intellectual disability, only one of whom had a potential seizure history, suggested a significant reduction in the incidence of non-affective aggression ${ }^{18}$. In 1993, an open prospective study involving use of VPA to treat behavioral problems in patients with intellectual disability $(n=18)$ found that $78 \%$ of patients responded favorably to treatment ${ }^{19}$. In this study, a favorable response to treatment was especially associated with a history of epilepsy or seizure. A retrospective study in 1999 investigating the effect of divalproex sodium (a compound of sodium valproate and valproic acid) on aggression in adults with intellectual disability $(n=28)$ found that a majority of treated patients exhibited significant reductions in $\operatorname{aggression}^{20}$. A retrospective study of divalproex sodium in 14 patients with DSM-IV Autism, Asperger's, or PDD NOS noted global improvement in various measures of ASD (including aggression) in $71 \%(\mathrm{n}=10)$ of patients ${ }^{21}$. In this study, all patients with a history of a seizure disorder or abnormal EEG readings were in the responder category. In 2005, a prospective double-blind placebo-controlled study which randomized 30 patients with pervasive neurodevelopmental disorder to treatment or placebo failed to identify a treatment difference between VPA and placebo groups ${ }^{22}$. Notably, this study excluded patients with a history of recent seizures or prior VPA trials. In 2010, a randomized, double-blind, placebo-controlled study involving 55 ASD patients found that $63 \%$ of VPA but only $9 \%$ of placebo cases 
responded with significant improvements in irritability, with a dose-dependent trend as assessed by VPA levels ${ }^{23}$. Subjects with "active or unstable epilepsy" were excluded. Overall, the preponderance of studies indicate that VPA may be effective for ASD patients with irritability. This finding is consistent with the pattern of treatment and symptom response observed in the case described in this report.

The patient evinced an array of symptomatology, but most prominently a disorganized and agitated affect, hallucinations with reactivity to internal stimuli and profound irritability. These features led to an initial conceptualization of primary psychosis. However, the totality of his clinical presentation, which included degraded sleep, distractibility, pressured mannerisms, and clear periodicity - which is to say, his symptoms were episodic and loosely circumscribed by initial and terminal boundaries beyond which the patient appeared to be at his baseline - has led to a mixed-state mania as the most favorable diagnostic conceptualization of this patient's case. There is literature to suggest that rates of bipolar disorder are significantly elevated in ASD. Specifically, the studies below indicate the importance of including a bipolar disorder on the differential when treating ASD patients with episodic irritability with affective dysregulation.

A study involving consecutive evaluation of patients referred to a pediatric psychopharmacology clinic showed that $21 \%(\mathrm{n}=14)$ of patients with a DSM-III-R diagnosis of autism, pervasive development disorder (PDD) and/or PDD NOS also met criteria for mania ${ }^{24}$. In a follow up study, $30 \%(n=47)$ of patients with bipolar 1 disorder also met criteria for an $\mathrm{ASD}^{25}$. Both of the aforementioned studies involved children and adolescents only. In a study investigating ASD comorbidities in adult patients, 7\% $(n=9)$ of study subjects with ASD also met criteria for bipolar disorder with psychotic features ${ }^{26}$. Finally, a systematic review investigating the comorbidity of bipolar disorder with Asperger's syndrome (a high functioning ASD) estimated a comorbidity prevalence ranging from 6-21\% ${ }^{9}$. All of these estimates are significantly higher than the approximate $1 \%$ prevalence of bipolar disorder in the general population ${ }^{27}$. Additionally, manias in the context of comorbid ASD may be significantly more likely to present in a mixed (dysphoric/aggressive) state relative to the euphoric affect classically associated with this condition, contributing to the potential for this diagnosis to be missed when comorbid in ASD individuals ${ }^{28}$. The diagnostic conceptualization of the patient's case as one with comorbid ASD and bipolar disorder is also consistent with the patient's history of depression (often a historic affective precursor to frank mania) and the improvement of his symptomatology with increase of his valproic acid dose, in conjunction with aggressively titrated atypical antipsychotic medication. 


\section{CONCLUSION}

Multiple diagnoses were considered in this complex patient. Bipolar disorder with irritable mania was ultimately favored as the most likely diagnosis. Bipolar disorder may be more common in patients with autism spectrum disorder. Early treatment with mood stabilizing anticonvulsants should be considered in ASD patients presenting with severe affective dysregulation, particularly when there is a history of epilepsy.

\section{AUTHOR NOTE:}

I am a military service member or employee of the U.S. Government. This work was prepared as part of my official duties. Title 17, U.S.C. $§ 105$ provides that copyright protection under this title is not available for any work of the U.S. Government. Title 17, U.S.C. §101 defines a U.S. Government work as work prepared by a military service member or employee of the U.S. Government as part of that person's official duties.

"The views expressed in this article reflect the results of research conducted by the author and do not necessarily reflect the official policy or position of the Department of the Navy, Department of Defense, nor the United States Government."

\section{REFERENCES}

1. Diagnotsic and Statistical Manual of Mental Disorders 5th ed. American Psychiatric Association. 2013;

2. Baxter A. J., Brugha T. S., Erskine H. E., Scheurer R. W., Vos T., Scott J. G.. The epidemiology and global burden of autism spectrum disorders. Psychological Medicine. 2015;45(3):601-613.

3. Besag Frank. Epilepsy in patients with autism: links, risks and treatment challenges. Neuropsychiatric Disease and Treatment. 2017; Volume 14:1-10.

4. Joshi Gagan, Petty Carter, Wozniak Janet, et al. The Heavy Burden of Psychiatric Comorbidity in Youth with Autism Spectrum Disorders: A Large Comparative Study of a Psychiatrically Referred Population. Journal of Autism and Developmental Disorders. 2010;40(11):1361-1370.

5. Kanne Stephen M., Mazurek Micah O.. Aggression in Children and Adolescents with ASD:

Prevalence and Risk Factors. Journal of Autism and Developmental Disorders. 2011;41(7):926-937. 
6. Posey David J., Stigler Kimberly A., Erickson Craig A., McDougle Christopher J.. Antipsychotics in the treatment of autism. Journal of Clinical Investigation. 2008;118(1):6-14.

7. Accordino Robert E., Kidd Christen, Politte Laura C., Henry Charles A., McDougle Christopher J.. Psychopharmacological interventions in autism spectrum disorder. Expert Opinion on Pharmacotherapy. 2016;17(7):937-952.

8. Golubchik Pavel, Sever Jonathan, Weizman Abraham. Low-Dose Quetiapine for Adolescents With Autistic Spectrum Disorder and Aggressive Behavior. Clinical Neuropharmacology. 2011;34(6):216-219.

9. Vannuchi G, Masi G, Tonic C. Bipolar disorder in adults with Asperger's Syndrome: a systematic review. J Affect Disord. 2014;168:151-60.

10. Stubbs Jean H, Hutchins David A, Mountjoy Christopher Q. Relationship of akathisia to aggressive and self-injurious behaviour: A prevalence study in a UK tertiary referral centre. International Journal of Psychiatry in Clinical Practice. 2000;4(4):319-325.

11. Research Johnson \&; Johnson Pharmaceutical, Development L L. A Study of the Efficacy and Safety of Topiramate Treatment in Patients with Epilepsy. Identifier: NCT00266591; 2005.

12. Crawford P. An Audit of topiramate use in a general neurology clinic. Seizure. JUN. 1998;7(3):207-211.

13. Khan Adil, Faught Edward, Gilliam Frank, Kuzniecky Ruben. Acute psychotic symptoms induced by topiramate. Seizure. 1999;8(4):235-237.

14. Cheung Eddie, Wong Virginia, Fung Cheuk-Wing. Topiramate-Valproate-Induced Hyperammonemic Encephalopathy Syndrome: Case Report. Journal of Child Neurology. 2005;20(2):157-160.

15. Chen Z, Lusicic A, Brien O, T . Psychotic disorders induced by antiepileptic drugs in people with epilepsy. Brain. ;2016(10):2668-2678.

16. Childs Judith A., Blair Joan L.. Valproic Acid Treatment of Epilepsy in Autistic Twins. Journal of Neuroscience Nursing. 1997;29(4):244-248.

17. Plioplys Audrius V.. Autism: Electroencephalogram Abnormalities and Clinical Improvement With Valproic Acid. Archives of Pediatrics \& Adolescent Medicine. 1994;148(2):220-220. 
18. Mattes J. Valproic Acid for Nonaffective Aggression in the Mentally Retarded. J Nerv Ment Dis. 1992;180(9):601-603.

19. KASTNER THEODORE, FINESMITH ROSS, WALSH KEVIN. Long-Term Administration of Valproic Acid in the Treatment of Affective Symptoms in People With Mental Retardation. Journal of Clinical Psychopharmacology. 1993;13(6):448???451-448???451.

20. Ruedrich S., Swales T. P., Fossaceca C., Toliver J., Rutkowski A.. Effect of divalproex sodium on aggression and self-injurious behaviour in adults with intellectual disability: a retrospective review. Journal of Intellectual Disability Research. 1999;43(2):105-111.

21. Hollander Eric, Dolgoff-Kaspar Rima, Cartwright Charles, Rawitt Ronald, Novotny Sherie. An Open Trial of Divalproex Sodium in Autism Spectrum Disorders. The Journal of Clinical Psychiatry. 2001;62(7):530-534.

22. Hellings J, Weckbaugh M, Nickel E, et al. A Double-Blind, Placebo-Controlled Study of Valproate for Aggression in Youth With Pervasive Developmental Disorders. J Child Adolesc Psychopharmacol. 2005;15(4):682-92.

23. Hollander Eric, Chaplin William, Soorya Latha, et al. Divalproex Sodium vs Placebo for the Treatment of Irritability in Children and Adolescents with Autism Spectrum Disorders. Neuropsychopharmacology. 2010;35(4):990-998.

24. Wozniak J, Biederman J, Faraone S, et al. Mania in Children with Pervasive Developmental Disorder Revisited.. J Am Acad Child Adolesc Psychiatry. 1997;36:1552-1561.

25. Joshi G, Biederman J, Petty C, Goldin R, Furtak S, Wozniak J. Examining the Comorbidity of Bipolar Disorder and Autism Spectrum Disorders: A Large Controlled Analysis of Phenotypic and Familial Correlates in a Referred Population of Youth with Bipolar I Disorder With and Without Autism Spectrum Disorders. J Clin Psychiatry. 2013;74(6):578-86.

26. Stahlberg O., Soderstrom H., Rastam M., Gillberg C.. Bipolar disorder, schizophrenia, and other psychotic disorders in adults with childhood onset AD/HD and/or autism spectrum disorders. Journal of Neural Transmission. 2004;111(7):891-902.

27. Belmaker R. H.. Bipolar Disorder. New England Journal of Medicine. 2004;351(5):476-486. 
28. Skeppar P, Thoor R, Agren S. Neurodevelopmental Disorders with Comorbid Affective Disorders Sometimes Produce Psychiatric Conditions Traditionally Diagnosed as Schizophrenia. Clin Neuropsychiatry. ;2013(10):123-156. 